\title{
Lévy Processes in Gold Option Modeling
}

\author{
Sandya N. Kumari ${ }^{1}$ \\ ${ }^{1}$ Department of Science and Technology, Faculty of Applied Sciences, Uva Wellassa University, Sri Lanka \\ Correspondence: Sandya N. Kumari, Department of Science and Technology, Faculty of Applied Sciences, Uva \\ Wellassa University, Sri Lanka. Tel: 94-711-244-919. E-mail: sandya@uwu.ac.lk
}

Received: December 3, 2019

Accepted: January 9, 2020

Online Published: January 29, 2020

doi:10.5539/ijef.v12n2p65

URL: https://doi.org/10.5539/ijef.v12n2p65

\begin{abstract}
To price and hedge derivative securities, it is crucial to have a good model of the probability distribution of the underlying product. In financial markets under uncertainty, the classical Black-Scholes model cannot explain the empirical facts. To overcome this drawback, the Lévy process was introduced to financial modeling. Today Gold futures markets are highly volatile. The purpose of this paper is to develop a mathematical framework in which American options on Gold futures contracts are priced more effectively. In this work, the Generalized Hyperbolic process, Normal Inverse Gaussian Process, Generalized Inverse Gaussian Process and Variance Gamma Process were used to model the future price. Then, option prices under the risk-neutral pricing process were calibrated and then authors attempt to infer the density forecast of future Gold prices at a given time horizon. Finally, Normal Inverse Gaussian was selected as the best model for Gold options by significant quantitative comparison between parsimonious models.
\end{abstract}

Keywords: financial market, lévy process, gold option, volatile

\section{Introduction}

Modeling the dynamics of financial asset prices is the core of modern finance theory. To price and hedge derivative securities, it is crucial to have a good model of the probability distribution of the underlying product. The most famous continuous-time model is the celebrated Black-Scholes model, which uses the Normal distribution to fit the log-returns of the underlying securities. As shown in previous studies, one of the main problems with the BlackScholes model (Black \& Scholes, 1973) is that the log-returns of stocks/indices are not normally distributed in real-life. The log-returns of most financial assets do not follow the Normal law (Cont, 2001; Heyde \& Leonenko, 2005; Kumari \& Tan, 2013). They are skewed and have an actual kurtosis higher than that of the normal distribution. Furthermore, in traditional diffusion models, price movements are very small in a short period of time. However, in real markets, prices may show big jumps in short time periods. When the price process model includes jumps, the perfect hedging is impossible. In this case, market participants cannot hedge risks by using only underlying assets. For these reasons, diffusion models used in finance is not a sufficient model.

Therefore, other more flexible distributions are needed. Moreover, it is required to have flexible stochastic processes with generalizing the Brownian motion. In order to define a stochastic process with independent increments, the distribution has to be infinitely divisible. Such processes are called Lévy processes. The term "Lévy process" honors the work of the French mathematician Paul Lévy who played an instrumental role in bringing together an understanding and characterization of processes with stationary independent increments. Lévy processes form a central class of stochastic processes, containing both Brownian motion and the Poisson process, and are prototypes of Markov processes and semi-martingales. Like Brownian motion, they are used in a multitude of applications ranging from biology and physics to insurance and finance. This continues to motivate further research in both theoretical and applied fields of finance (Eberlein, 2007) and their application appears in the theory of many areas of classical and modern stochastic processes of finance and other fields (Kyprianou, 2014).

'Gold' has captured the imagination for thousands of years. Yet, despite the growing importance of derivatives, relatively little research has been done on the gold options market. Therefore, the purpose of this study is to develop the best option pricing formula for Gold future options which are traded on the Chicago Mercantile Exchange (COMEX) under Lévy process frameworks more effectively than Brownian Motion. According to our knowledge, this is the first study that introduces the option pricing formula for a Gold option based on the Lévy process. To fulfill the objective, the pure jumped Lévy processes: the Generalized Hyperbolic model, Normal Inverse Gaussian 
process, Generalized Inverse Gaussian process, and variance Gamma process were focused. Finally, option pricing based on pure-jumped Lévy models were calibrated with application to Gold options and attempts to infer the density forecast of future Gold prices at a given time horizon. Furthermore, the gain of jump Lévy models over the Black-Scholes model was judged.

\section{Lévy Processes}

\subsection{Mathematical Background}

Stochastic processes are collections of random variables $X_{t}, t \geq 0$ (meaning $t \in[0, \infty$ ) as opposed to $n \geq 0$ by which means $n \in \mathbb{N}=\{0,1,2, \ldots\}$ ). For us, all $X_{t}, t \geq 0$, take values in a common state space, which we will choose specifically as $X_{t}, \mathbb{R}\left(\right.$ or $[0, \infty)$ or $\mathbb{R}_{d}$ for some $d \geq 2$ ). $X_{t}$ as the position of a particle at time $t$, changing as $t$ varies. It is natural to suppose that the particle moves continuously in the sense that $t \mapsto X_{t}$ is continuous (with probability 1 ), or that it has jumped for some $t \geq 0$ :

$$
\triangle X_{t}=X_{t+}-X_{t-}=\lim _{\varepsilon \downarrow 0} X_{t+\varepsilon}-\lim _{\varepsilon \downarrow 0} X_{t-\varepsilon} .
$$

It is usually assumed that these limits exist for all $t \geq 0$ and that in fact $X_{t+}=X_{t}$, i.e. that $t \mapsto X_{t}$ is rightcontinuous with left limits $X_{t-}$ for all $t \geq 0$ almost surely. The path $t \mapsto X_{t}$ can then be viewed as a random right-continuous function.

Definition 1: A stochastic process $X=\left(X_{t}\right)_{t \geq 0}$ defined on a probability space $(\Omega, \mathcal{F}, P)$, taking values in $\mathbb{R}$, is said to be a Lévy process if it possesses the following properties:

$i$. The paths of $X$ are $P$-almost surely right continuous with left limits. (ie. cadlag paths)

ii. $P\left(X_{0}=0\right)=1$

iii. X has independent increments of the past i.e. $X_{t}-X_{s}$ independent of $\left\{X_{u}: u \leq s\right\}$ for $0 \leq s \leq t$

$i v$. X has stationary increments i.e $X_{t}-X_{s}$ has the same distribution with $X_{t-s}$

v. X is stochastically continuous, $\lim _{k \rightarrow \infty} P\left(\left|X_{t+k}-X_{t}\right| \geq \varepsilon\right), \forall \varepsilon>0$

The most commonly known examples of Lévy processes are the Poisson process and Brownian motion (also named Wiener process). Furthermore, De Finetti (1929) introduced the notion of an infinitely divisible distribution and showed that they have an intimate relationship with Lévy processes. This relationship gives a reasonably good impression of how varied the class of Lévy processes really is.

Definition 2: A real-valued random variable $X$ has an infinitely divisible distribution if for each $n=1,2, \ldots$ there exist a sequence of independently identical distribution (i.i.d) random variables $X_{1, n}, X_{2, n}, \ldots . X_{n, n}$ such that,

$$
X^{\stackrel{d}{=}} X_{1, n}+X_{2, n}+\ldots .+X_{n, n}
$$

where $\stackrel{a}{=}$ is equality in distribution. Alternatively, we could have expressed this relation in terms of probability laws. That is to say, the law $\mu$ of a real-valued random variable is infinitely divisible if for each $n=1,2, \ldots$ there exists another law $\mu_{n}$ of a real-valued random variable such that $\mu=\mu_{n}^{* n}$. (Here $\mu_{n}^{* n}$ denotes the $n$-fold convolution of $\mu_{n}$ ).

In view of the above definition, one way to establish whether a given random variable has an infinitely divisible distribution is via its characteristic exponent. Suppose that $X$ has characteristic exponent $\Psi(u):=\log \mathbb{E}\left[e^{-i u x}\right]$ for all $u \in \mathbb{R}$. Then $X$ has an infinitely divisible distribution if for all $n \geq 1$ there exists a characteristic exponent of a probability distribution, say $\Psi_{n}$, such that $\Psi(u)=n \Psi_{n}(u)$ for all $u \in \mathbb{R}$.

Many known distributions are infinitely divisible however some are not. As examples, the Normal, Poisson, Gamma and geometric distributions are infinitely divisible. This often follows from the closure under convolutions of the type:

$$
\begin{gathered}
Y_{1} \sim \operatorname{Normal}\left(\mu_{1}, \sigma_{1}^{2}\right) \& Y_{2} \sim \operatorname{Normal}\left(\mu_{2}, \sigma_{2}^{2}\right) \Rightarrow \\
Y_{1}+Y_{2} \sim \operatorname{Normal}\left(\mu_{1}+\mu_{2}, \quad \sigma_{1}^{2}+\sigma_{2}^{2}\right)
\end{gathered}
$$

for independent $Y_{1}$ and $Y_{2}$ since this implies by induction that for independent

$$
\begin{gathered}
\mathrm{Y}_{1}^{(\mathrm{m})}, \mathrm{Y}_{2}^{(\mathrm{m})}, \ldots, \mathrm{Y}_{\mathrm{n}}^{(\mathrm{m})} \sim \operatorname{Normal}\left(\mu / \mathrm{m}, \sigma^{2} / \mathrm{m}\right) \Rightarrow \\
\mathrm{Y}_{1}^{(\mathrm{m})}+\mathrm{Y}_{2}^{(\mathrm{m})}+\ldots+\mathrm{Y}_{\mathrm{n}}^{(\mathrm{m})} \sim \operatorname{Normal}\left(\mu, \sigma^{2}\right) .
\end{gathered}
$$

The full extent to which may characterize infinitely divisible distributions is described by the characteristic 
exponent $\Psi$ and an expression known as the Lévy-Khintchine formula.

Theorem 1: (Lévy-Khintchine formula). A probability law $\mu$ of a real-valued random variable is infinitely divisible with characteristic exponent $\Psi$,

$$
\int_{\mathbb{R}} e^{i \theta x} \mu(d x)=e^{-\Psi(\theta)}, \theta \in \mathbb{R}
$$

iff there exists a triple $(a, \sigma, \Pi)$, where $a \in \mathbb{R}, \sigma \geq 0$ and $\Pi$ is a measure concentrated on $\mathbb{R} \backslash\{0\}$ satisfying $\int_{\mathbb{R}}(1 \wedge x) \Pi(d x)<\infty$, such that

$$
\Psi(\theta)=i a \theta+\frac{1}{2} \sigma^{2} \theta^{2}+\int_{\mathbb{R}}\left(1-e^{i \theta x}+i \theta x 1_{(|x|<1)}\right) \Pi(d x),
$$

for every $\theta \in \mathbb{R}$. The measure $\Pi$ is called the Lévy (characteristic) measure. Thus, $\Pi(d x)$ is the intensity of jumps of size $x$ and the function $\Psi(\theta)$ is called the Lévy exponent (or Lévy symbol).

From the definition of a Lévy process, we see that for any $t>0, X_{t}$ is a random variable belonging to the class of infinitely divisible distributions. This follows from the fact that for any $n=1,2, \ldots$,

$$
X_{t}=X_{t / n}+\left(X_{2 t / n}-X_{t / n}\right)+\ldots+\left(X_{t}-X_{(n-1) t / n}\right)
$$

together with the fact that $X$ has stationary independent increments. Suppose to define for all $\theta \in \mathbb{R}, t \geq 0$, $\Psi_{t}(\theta):=\log \mathbb{E}\left[e^{-i \theta x_{t}}\right]$, using $(01)$ twice we have for any two positive integers $\mathrm{m}, n$ that;

$$
\mathrm{m} \Psi_{\mathrm{t}}(\theta)=\Psi_{\mathrm{m}}(\theta)=n \Psi_{\mathrm{m} / \mathrm{n}}(\theta)
$$

and hence for any rational $t>0$,

$$
\Psi_{t}(\theta)=t \Psi_{1}(\theta)
$$

If $t$ is an irrational number, by choosing a decreasing sequence of rational $\left\{t_{n}: n \geq 1\right\}$ such that $t_{n} \downarrow t$ as $n \rightarrow$ $\infty$. Almost sure right continuity of $X$ implies right continuity of $\exp \left\{-\Psi_{\mathrm{t}}(\theta)\right\}$ (by dominated convergence) and hence (02) holds for all $t \geq 0$. In conclusion, any Lévy process has the property that for all $t \geq 0$,

$$
\mathbb{E}\left[e^{i \theta X_{t}}\right]=e^{-t \psi(\theta)},
$$

where $\Psi(\theta):=\Psi_{1}(\theta)$ is the characteristic exponent of $X_{1}$, which has an infinitely divisible distribution.

\subsection{Self-Decomposability}

A random variable (r.v.) $X$ is self decomposable if its characteristic function $\Psi(u)$ has the property that, there exist a characteristic function $\Psi_{\mathrm{c}}(u)$, for every $0<\mathrm{c}<1$ such that;

$$
\Psi(u)=\Psi(c u) \Psi_{c}(u) \text {; for all } u \in \mathbb{R} .
$$

In term of the random variable $X$ the above means that for any $0<\mathrm{c}<1$ there exits a r.v. $X_{c}$ such that;

$$
X \stackrel{a}{=} c X+X_{c}
$$

with independent r.v. $X$ and $X_{c}$, where $\stackrel{a}{=}$ means equality in distribution.

A random variable $X$ has a distribution of class $L$ (Note 1) iff the law of the $X$ is self-decomposable. The class of self-decomposable distribution is a subclass of infinitely divisible distributions. Self-decomposable laws arise as marginal laws in autoregressive time series models as: $X_{t}=c X_{t-1}+\varepsilon_{t}$. The Lévy measure of the selfdecomposable laws is absolutely continuous with the following density form,

$$
\Pi(d x)=k(x) d x
$$

where $k(x)$ is a Lévy density which has the same mathematical requirements as a probability density. Further, $|x| k(x)$ is increasing for $(-\infty, 0)$ and decreasing for $(0, \infty)$. The density of self-decomposable distributions is unimodal.

Let be a Lévy process $X=\left(X_{t}: t \geq 0\right)$. $\left(\mathrm{X}_{1}\right)$ is self-decomposable iff $\left(\mathrm{X}_{\mathrm{t}}\right)$ is self-decomposable for every $\mathrm{t}>$ 0 (Carr, Geman, Madan and Yor (2007)). The characteristic function of self-decomposable laws has the following form,

$$
\Psi(\theta)=i a \theta+\frac{1}{2} \sigma^{2} \theta^{2}+\int_{\mathbb{R}}\left(1-e^{i \theta x}+i \theta x 1_{(|x|<1)}\right) \frac{k(x)}{x} d x
$$

where $\theta \in \mathbb{R}, k(x) \geq 0$ and $\int_{\mathbb{R}} \min \left(1,|x|^{2}\right) \frac{\mathrm{k}(\mathrm{x})}{\mathrm{x}} \mathrm{dx}<\infty$, (Sato(1999), p.95, Corollary15.11), (Carr et al. (2007, p. 34). A self-decomposable random variable $X$ is the value at the unit time of some pure jump Lévy 
processes in which sample paths have bounded variation. When the Lévy density integrates $|X|$ in the region $|X|<1$, for $\theta=\int_{|x|<1} x \frac{\mathrm{k}(\mathrm{x})}{\mathrm{x}} \mathrm{dx}$. The characteristic function of the processes $X$,

$$
E\left[e^{i u x}\right]=\exp \left[\int_{-\infty}^{\infty} e^{i u x_{1}} \frac{k(x)}{x} d x\right] .
$$

Consider that, the returns are the sum of a suitable number of approximately independent random variables and return distribution is a limit distribution. Self-decomposable distributions can be considered as a candidate for the unit period distribution of asset returns. Halgreen (1977) is shown that hyperbolic distributions are selfdecomposable.

\subsection{Self-similarity and Self-Decomposability}

A stochastic process $\mathrm{X}=\left(\mathrm{X}_{\mathrm{t}}: t \geq 0\right)$ is called self-similar for any given $c \geq 0$,

$$
\left(\mathrm{X}_{\mathrm{ct}}: t \geq 0\right) \stackrel{d}{=}\left(\mathrm{c}^{\mathrm{H}} \mathrm{X}_{\mathrm{t}}: t \geq 0\right)
$$

where, $H>0$ is Hurst exponent Petroni ((2008), p.1882). In other words, one stochastic process is self-similar such that, the change in time scale can be compensated by a corresponding change in the scale of the spaces. The connection between self-decomposable laws and the self-similar additive process is given by Sato (1991). Law is self-decomposable if and only if it is the law at the unit time of a self-similar additive process. Let $\Psi(\theta)$ be a characteristic function of law, then, it can take a characteristic function of the Lévy process as,

$$
\Psi_{t}(\theta)=[\Psi(\theta)]^{t / N}
$$

where, $N$ is time scale, if $\Psi(\theta)$ is infinite divisible, $\Psi_{t}(\theta)$ is a characteristic function. New function describes as,

$$
\varphi_{k, H}(\theta)=\Psi\left[\left(\frac{k}{N}\right)^{H} \theta\right] \Psi\left[\left(\frac{k}{N}\right)^{H} \theta\right]^{-1} .
$$

It is a characteristic function if and only if $\Psi(u)$ is self-decomposable by Sato (1999, p. 99), Petroni (2008, p. 1884). The stationary process and the self-similar process are related by using the first Lamperti representation (Aballero \& Chaumont, 2006).

\subsection{Subordination}

Subordination is a way of constructing new Lévy processes from existing ones. More preciously, subordinator is a one-dimensional stochastic process that is non-decreasing almost surely. Let $G$ be a Lévy process with Lévy exponent $\varphi(u)$ and let $W$ be a Lévy process with Lévy exponent $l(u)$, then $X$ be a subordinated process which can be expressed as; $X_{t}=l_{G_{t}}$.

Theorem 3.2: Let $G$ be a Lévy process with Lévy exponent $\varphi(u)$, and let $W$ be a Lévy process and subordinator with Lévy exponent $l(u)$. Then the process $X=\left\{X_{t}, t \geq 0\right\}$ defined for each $\omega \epsilon \Omega$ by $X(t, \omega)=$ $G(W(t, \omega), \omega)$ is a Lévy process with characteristic function given by;

$$
\phi_{t}(u, t)=\exp \{t l(-i \varphi(u))\}
$$

ie. The Lévy exponent of $X$ is given as a composition of the Lévy exponent of $G$ and $W$.

\section{Lévy Process Frameworks}

Under this section, pure jump Lévy processes are presented in which the asset price dynamics are modeled by the pure jump Lévy process. This means that the asset price moves only by jumps with zero Gaussian variance in Lévy-Khintchine formula. The generalized hyperbolic process, normal inverse Gaussian process, generalized inverse Gaussian process and variance gamma process are discussed under characteristic function, Lévy process and together with some more properties.

\subsection{Generalized Hyperbolic (GH) Processes}

At present, many researches regarding the modeling of financial assets are focused on the market model based on the Generalized Hyperbolic Distribution (N. Ciprian 2009). The PDF of the GH distribution can be defined by:

$$
\mathrm{f}_{\mathrm{GH}}(\mathrm{x} ; \lambda, \alpha, \beta, \delta, \mu)=\mathrm{a}(\lambda, \alpha, \beta, \delta)\left(\delta^{2}+(\mathrm{x}-\mu)^{2}\right)^{\frac{(\lambda-1 / 2)}{2}} \mathrm{~K}_{\lambda-1 / 2}\left(\alpha \sqrt{\delta^{2}+(\mathrm{x}-\mu)^{2}}\right) \mathrm{e}^{(\beta(\mathrm{x}-\mu))}
$$

where, $\mathrm{a}(\lambda, \alpha, \beta, \delta)=\frac{\left(\alpha^{2}-\beta^{2}\right)^{\lambda / 2}}{\sqrt{2 \pi} \alpha^{\lambda-1 / 2} \delta^{\lambda} \mathrm{K}_{\lambda}\left(\sqrt{\alpha^{2}-\beta^{2}}\right)}$ is the normalizing constant and $\mathrm{K}_{\nu}$ denotes the modified Bessel function of the third kind with index $v$. An integral representation of $K_{v}$ is given by; $K_{v}(z)=$ 
$\frac{1}{2} \int_{0}^{\infty} \mathrm{y}^{\mathrm{v}-1} \exp \left(-\frac{1}{2} \mathrm{z}\left(\mathrm{y}+\mathrm{y}^{-1}\right)\right) \mathrm{dy}$

Generalized hyperbolic distributions have a number of appealing analytic properties. Their moment generating function is given by;

$$
M_{G H}(\mathrm{u})=\mathrm{e}^{\mu \mathrm{u}}\left(\frac{\alpha^{2}-\beta^{2}}{\alpha^{2}-(\beta+u)^{2}}\right)^{\lambda / 2} \frac{K_{\lambda}\left(\delta \sqrt{\alpha^{2}-(\beta+u)^{2}}\right)}{K_{\lambda}\left(\delta \sqrt{\alpha^{2}-\beta^{2}}\right)}, \text { for }|\beta+u|<\alpha
$$

where $K_{\lambda}(x)$ denotes the modified Bessel function of the third kind with index $\lambda$. From this formula, moments of any integer order can be derived. The first moment or mean can be defined as $E[G H]=\mu+\frac{\beta \delta^{2}}{\omega} \frac{K_{\lambda+1}(\omega)}{K_{\lambda}(\omega)}$ and for the variance: $V[G H]=\frac{\delta^{2}}{\omega} \frac{K_{\lambda+1}(\omega)}{K_{\lambda}(\omega)}+\frac{\beta^{2} \delta^{4}}{\omega^{2}}\left(\frac{K_{\lambda+2}(\omega)}{K_{\lambda}(\omega)}-\frac{K_{\lambda+1}^{2}(\omega)}{K_{\lambda}^{2}(\omega)}\right)$ with $=\delta \sqrt{\alpha^{2}-\beta^{2}}$. Note that these expressions simplify considerably in the case of symmetry, i.e. for $\beta=0$. Also the characteristic function $\phi_{G H}$ is easily obtained by exploiting the relation $\phi_{G H}(u)=M_{G H}(\mathrm{iu})$ and given by,

$$
\phi_{G H}(u ; \lambda, \alpha, \beta, \delta, \mu)=\mathrm{e}^{i u \mu}\left(\frac{\alpha^{2}-\beta^{2}}{\alpha^{2}-(\beta+i u)^{2}}\right)^{\lambda / 2} \frac{K_{\lambda}\left(\delta \sqrt{\alpha^{2}-(\beta+i u)^{2}}\right)}{K_{\lambda}\left(\delta \sqrt{\alpha^{2}-\beta^{2}}\right)} .
$$

The Lévy-Khintchine representation of the characteristic function of generalized hyperbolic distributions is given by;

$$
\ln \left(\phi_{G H}(u)\right)=i u E[G H]+\int_{-\infty}^{\infty}\left(e^{i u x}-1-i u x\right) g(x) d x
$$

where the density $g(x)$ of the Lévy measure has the following form;

$$
g(x)=\left\{\begin{array}{ll}
\frac{e^{\beta x}}{|x|}\left(\int_{0}^{\infty} \frac{\exp \left(-\sqrt{2 y+\alpha^{2}}|x|\right)}{\pi^{2} y\left(J_{\lambda}^{2}(\delta \sqrt{2 y})+Y_{\lambda}^{2}(\delta \sqrt{2 y})\right)} d y+\lambda e^{\alpha|x|}\right) & \text { if } \lambda \geq 0 \\
\frac{e^{\beta x}}{|x|} \int_{0}^{\infty} \frac{\exp \left(-\sqrt{2 y+\alpha^{2}}|x|\right)}{\pi^{2} y\left(J_{-\lambda}^{2}(\delta \sqrt{2 y})+Y_{-\lambda}^{2}(\delta \sqrt{2 y})\right)} d y & \text { if } \lambda<0
\end{array} .\right.
$$

Here $J_{\lambda}$ and $Y_{\lambda}$ are the Bessel functions of the first and second kind with index $\lambda$, respectively. A characteristic of the GH process refers to the fact that it has no diffusion component. Therefore, the GH process is a "pure jump" process. The GH distribution turns out to be infinitely divisible and we can be defined as a GH Lévy process such that $X^{(G H)}=\left\{X_{t}^{(G H)}, t \geq 0\right\}$ as the stationary process starts at zero and has an independent increment. The distribution of $X_{t}^{(G H)}$ has a characteristic function, $E\left[\exp \left(i u X_{t}^{(G H)}\right)\right]=\left(\phi_{G H}(u ; \lambda, \alpha, \beta, \delta, \mu)\right)^{t}$ that means $X_{1} \sim G H(\lambda, \alpha, \beta, \delta, \mu)$.

\subsection{Normal Inverse Gaussian (NIG) Process}

The NIG distribution with parameters $\alpha>0,(\beta \in(-\alpha, \alpha), \delta>0$ and $\mu>0, N I G(\alpha, \beta, \delta, \mu)$, has a characteristic function[20];

$$
\phi_{N I G}(u)=\mathrm{e}^{i u \mu} \frac{\exp \left(\delta \sqrt{\alpha^{2}-\beta^{2}}\right)}{\exp \left(\delta \sqrt{\alpha^{2}-(\beta+i u)^{2}}\right)}
$$

This is an infinitely divisible characteristic function (Barndorff-Nielsen, O. E., 1995). Hence, the NIG Lévy process can define as; $X^{(N I G)}=\left\{X_{t}^{(N I G)}, t \geq 0\right\}$, with $X_{0}^{(N I G)}=0$, stationary and independent NIG distributed increments. To be precise, $X_{t}^{(N I G)}$, has a $N I G(\alpha, \beta, \delta t, \mu t)$ law with the characteristic function of the form $\left(\phi_{N I G}(u)\right)^{t}$. The Lévy-Khintchine representation of NIG is given by;

$$
\ln \left(\phi_{N I G}(u)\right)=i u E[N I G]+\int_{-\infty}^{\infty}\left(e^{i u x}-1-i u x\right) g(x) d x
$$

where the density $g(x)$ of the Lévy measure has the following form; $g(x)=e^{\beta x} \frac{\alpha \delta}{\pi|x|} K_{1}(\alpha|x|)$, where $K_{\lambda}(x)$ 
denotes the modified Bessel function of the third kind with index $\lambda$. A NIG process has no Brownian component. Therefore, the NIG process is a "pure jump" process.

The first and second moments of the NIG distribution are

$$
E[N I G]=\mu+\frac{\beta \delta}{\sqrt{\alpha^{2}-\beta^{2}}} \text { and } V[N I G]=\frac{\delta}{\sqrt{\alpha^{2}-\beta^{2}}}+\frac{\beta^{2} \delta}{\left(\sqrt{\alpha^{2}-\beta^{2}}\right)^{3}} .
$$

The NIG is the only subclass of the GH that is closed under convolution, i.e. if $X_{1} \sim N I G\left(\alpha, \beta, \delta_{1}, \mu_{1}\right)$ and $X_{2} \sim N I G\left(\alpha, \beta, \delta_{2}, \mu_{2}\right)$ and $X_{1}$ is independent of $X_{2}$, then $X_{1}+X_{2} \sim N I G\left(\alpha, \beta, \delta_{1}+\delta_{2}, \mu_{1}+\mu_{2}\right)$. Therefore, if we estimate the returns distribution at some time scale, then we know it in closed form for all time scales. The NIG process is relatively straightforward to apply and can be simulated directly, which will be more useful for pricing barrier options (Marc Cassagnoly, 2010).

\subsection{Generalized Inverse Gaussian (GIG) Process}

The Inverse Gaussian $\operatorname{IG}(\delta, \gamma)$ (Note 2) law can be generalized to what is called the generalized inverse Gaussian distribution $\operatorname{GI} G(\lambda, \delta, \gamma)$. The distribution is given on the positive half-line;

$$
f_{G I G}(x ; \lambda, \delta, \gamma)=\left(\frac{\gamma}{\delta}\right)^{\lambda} \exp \left(-\frac{1}{2}\left(\frac{\delta^{2}}{x}+\gamma^{2} x\right)\right) \frac{1}{2 K_{\lambda}(\delta \gamma)} x^{\lambda-1} \text { for } x>0
$$

with the following parameter space;

$$
\begin{array}{ll}
\delta \geq 0, \gamma>0 & \text { if } \lambda>0 \\
\delta>0, \gamma>0 & \text { if } \lambda=0 \\
\delta>0, \gamma \geq 0 & \text { if } \lambda<0
\end{array}
$$

where $K_{\lambda}(x)$ denotes the modified Bessel function of the third kind with index $\lambda$. The characteristics function is given by;

$$
\phi_{G I G}(u)=\frac{1}{K_{\lambda}(\delta \gamma)}\left(1-\frac{2 i u}{\gamma^{2}}\right)^{\lambda / 2} K_{\lambda}\left(\delta \gamma \sqrt{1-2 i u \gamma^{-2}}\right) .
$$

Lévy-Khintchine representations of GIG distributions can be represented as;

$$
\phi_{G I G}(u)=\exp \left(i u \frac{\delta K_{\lambda+1}(\delta \gamma)}{\gamma K_{\lambda}(\delta \gamma)}+\int_{0}^{\infty}\left(e^{i u x}-1-i u x\right) g_{G I G}(x) d x\right)
$$

where the density of the Lévy measure is defined for $x>0$ by;

$$
g_{G I G}(x)=\frac{e^{-x \frac{\gamma^{2}}{2}}}{x}\left[\int_{0}^{\infty} \frac{\exp (-x y)}{\pi^{2} y\left(J_{|\lambda|}^{2}(\delta \sqrt{2 y})+Y_{|\lambda|}^{2}(\delta \sqrt{2 y})\right)} d y+\max (0, \lambda)\right],
$$

(Barndorff-Nielsen, 2001a), and where $J_{\lambda}$ and $Y_{\lambda}$ are Bessel functions.

Barndorff-Nielsen and Halgreen (1977) showed that the GIG distribution is infinitely divisible. Therefore, GIG process is defined as the Lévy process where the increment over the interval $[s, s+t], s, t \geq 0$, has the characteristic function $\left(\phi_{G I G}(u)\right)^{t}$.

\subsection{Variance Gamma (VG) Process}

The characteristic function of the $\operatorname{VG}(\theta, \sigma, v)$ law is given by;

$$
E\left[\exp \left(i u X_{t}\right)\right]=\phi_{V G}(u)=\left(1-i u \theta v+\frac{1}{2} \sigma^{2} v u^{2}\right)^{-\frac{1}{v}} .
$$

This distribution is an infinitely divisible and VG Lévy process can define as; $X^{(V G)}=\left\{X_{t}^{(V G)}, t \geq 0\right\}$, with $X_{0}^{(V G)}=0$, stationary and independent increments. The increment $X_{s+t}^{(V G)}-X_{t}^{(V G)}$ follows a $V G(t \theta, \sigma \sqrt{t}, v / t)$ law over the time interval $[s, s+t]$. Clearly $\left(s=0\right.$ and note that $\left.X_{0}^{(V G)}=0\right)$,

$$
E\left[\exp \left(i u X_{t}^{(V G)}\right)\right]=\phi_{V G}(u ; t \theta, \sigma \sqrt{t}, v / t)=\left(\phi_{V G}(u ; \theta, \sigma, v)\right)^{t}=\left(1-i u \theta v+\frac{1}{2} \sigma^{2} v u^{2}\right)^{-\frac{t}{v}}
$$

where the density function $X_{t}$ can be obtained as; 


$$
f_{V G}(x)=\frac{2 e^{\frac{\theta x}{\sigma^{2}}}}{v^{\frac{t}{v}} \sqrt{2 \pi} \sigma \Gamma\left(\frac{\mathrm{t}}{v}\right)}\left(\frac{x^{2}}{\frac{2 \sigma^{2}}{v}+\theta^{2}}\right)^{\frac{\mathrm{t}}{2 v}-\frac{1}{4}} K_{\frac{\mathrm{t}}{v}-\frac{1}{2}}\left(\frac{1}{\sigma^{2}} \sqrt{x^{2}\left(\frac{2 \sigma^{2}}{v}+\theta^{2}\right)}\right) .
$$

\section{Stock Price Models Driven by Lévy Processes}

Stock price behavior is now modeled by a more sophisticated stochastic process than the Brownian motion of the Black-Scholes model. That is an exponential Lévy process. There are no unique equivalent martingale measures (EMM) due to the fact that the Lévy models are incomplete. Therefore, a perfect hedge cannot be obtained when pricing options. In an options market, there are many different EMM under which the discounted asset price process is a martingale. The existence of a martingale measure is related to the absence of arbitrage, while the uniqueness of the equivalent martingale measure is related to market completeness. Let $X_{t}$ be a Lévy process on a filtered probability space $(\Omega, \mathcal{F}, P)$ with $P$ denoting the physical probability measure. Then assume that market consists of one riskless asset (the bond), with a price process given by $B_{t}=\exp (r t)$, and one risky asset (the stock or index). The risky asset model is given by: $S_{t}=S_{0} \exp \left(X_{t}\right)$. The log-returns $\log \frac{s_{t+s}}{s_{t}}$, of such a model follow the distribution of increments of length $\mathrm{s}$ of the Lévy process $X_{t}$.

\subsection{Equivalent Martingale Measure (EMM)}

An equivalent martingale measure or risk-neutral measure $Q$ is needed for valuation of the arbitrage-free price of the derivative product written on an asset with price process $S_{t}$, i.e. a probability measure $\mathrm{Q}$ is equivalent to $P$ under which the discounted price process $S_{t} \exp (-r t)$ evolves as a martingale. In this study, two ways of finding an EMM were considered: the Esscher transform martingale measure and a mean-correcting martingale measure.

\subsubsection{The Esscher Transform}

Following Gerber and Shiu (1994), we can find an Esscher transform of a stock-price process. Let $f_{t}^{(\theta)}(x)$ be the density of $\log$ price $\log S_{t}$ under $P$.

For some number $\theta \in\left\{\theta \in \mathbb{R} \mid \int_{-\infty}^{\infty} \exp (\theta y) f_{t}(y) d y<\infty\right.$, the new density is given by:

$$
f_{t}^{(\theta)}(x)=\frac{\exp (\theta x) f_{t}(x)}{\int_{-\infty}^{\infty} \exp (\theta y) f_{t}(y) d y} .
$$

Now choose $\theta$ such that the discounted price process $\left\{S_{t} \exp (-r t)\right\}_{t \geq 0}$ is a martingale, i.e.

$$
S_{0}=\exp (-r t) \mathbb{E}^{(\theta)}\left[S_{t}\right],
$$

where the expectation is taken with respect to the law with density $f_{t}^{(\theta)}$. Let $\phi(u)=\mathbb{E}\left[\exp \left(i u X_{1}\right)\right]$ denote the characteristic function of $X_{1}$. Hence, in order to let the discounted price process be a martingale, it is required to have,

$$
\exp (r)=\frac{\phi(-i(\theta+1))}{\phi(-i \theta)}
$$

The solution of this equation, $\theta^{*}$ say, gives the Esscher transform martingale measure through the density function $f_{t}^{\left(\theta^{*}\right)}(x)$. An alternative approach for computing a risk-neutral measure, similar to the Esscher transform, can also be proposed (Carr et al., 2003). Let $\left(X_{t}\right)_{t \geq 0}$ be a real-valued process with independent increments. Then $\left(\frac{\exp \left(i u X_{t}\right)}{\mathbb{E}\left[\exp \left(i u X_{t}\right)\right]}\right)_{t \geq 0}$ is a martingale $\forall u \in R$.

The asset price $S_{t}$ is modeled as $S_{t}=S_{0} \exp \left[r t+X_{t}\right]$ where $X_{t}$ is a Lévy process. Then, the resulting riskneutral process for the log price is given by;

$$
\log S_{t}=\log S_{0}+r t-\log \mathbb{E}\left[\exp \left(X_{t}\right)\right]+X_{t},
$$

and the Characteristic function of the log price is given by;

$$
\mathbb{E}\left[\exp \left(i u \log S_{t}\right)\right]=\exp \left(i u\left(\log S_{0}+r t-\log \mathbb{E}\left[\exp \left(X_{t}\right)\right]\right)\right) \mathbb{E}\left[\exp \left(i u X_{t}\right)\right] .
$$

\subsubsection{Mean-correcting Martingale Measure}

Another way to obtain an EMM is hat considering mean correcting the exponential of Lévy with reference special parameter $m$ (Note 3). First, all the parameters have to be estimated which are involved in the asset price process 
$S_{t}$, then the $\mathrm{m}$ parameter is changed in an appropriate way such that discounted asset price process becomes a martingale.

Let, specify,

$$
m_{\text {new }} \text { s.t. } m_{\text {new }}=m_{\text {old }}+r-q-\log \phi(-i) \text {, }
$$

where $\phi(x)$ is the characteristic function of the log return involving the $m_{\text {old }}$ parameter and $\mathrm{q} \in \mathbb{R}$ for the dividend rate. The choice of the $m_{\text {new }}$ imply that our discounted asset price $\mathbb{E}^{Q}\left[S_{t}\right]=S_{0} \exp [t(r-q)]$ is a martingale.

The $m_{\text {new }}$ parameters for different models are specified in Table 3.1 and it can be expected as;

i. Estimate the parameters of the process by fixing $m$ as 0

ii. Then introduce a parameter $m$ as an estimated parameters

Furthermore, $m_{\text {new }}$ can be considered as a risk-neutral $(\mathrm{RN})$ drift of a given process and it is as $\Delta$.

Table 1. The $\mathrm{m}$ parameter for the mean-correcting equivalent martingale measure

\begin{tabular}{lc}
\hline Model & $\boldsymbol{m}_{\text {new }}$ \\
\hline Normal & $r-q-\frac{1}{2} \sigma^{2}$ \\
GH & $r-q-\log \left(\left(\frac{\alpha^{2}-\beta^{2}}{\alpha^{2}-(\beta+1)^{2}}\right)^{\lambda / 2} \frac{K_{\lambda}\left(\delta \sqrt{\alpha^{2}-(\beta+1)^{2}}\right)}{K_{\lambda}\left(\delta \sqrt{\alpha^{2}-\beta^{2}}\right)}\right)$ \\
NIG & $r-q+\delta\left(\sqrt{\alpha^{2}-(\beta+1)^{2}}-\sqrt{\alpha^{2}-\beta^{2}}\right)$ \\
GIG & $r-q+\left(1-\frac{2}{\gamma^{2}}\right)^{\frac{\lambda}{2}} \frac{K_{\lambda}\left(\delta \gamma \sqrt{1-2 \gamma^{-2}}\right)}{K_{\lambda}(\delta \gamma)}$ \\
VG & $r-q-\frac{1}{v} \log \left(1+v \theta-\frac{1}{2} \sigma^{2} v\right)$ \\
\hline
\end{tabular}

The RN characteristic functions are given in the following Table 2 for the above discussed models. Assume that dividend yield $q$ is ignored after that for derivation. (As an example, refer Appendix A, for detail derivation for Variance Gamma RN process. In the same manner rest of the RN characteristics functions can be derived.)

Table 2. The risk-neutral characteristics function for selected Lévy processes

\begin{tabular}{lc}
\hline Model & $\boldsymbol{\phi}_{R N}(\boldsymbol{u}) \quad($ RN Characteristics Function $)$ \\
\hline Normal & $\phi_{R N}^{B S}(u)=e^{i \Delta u t-\frac{1}{2} \sigma^{2} u^{2} t}$ \\
GH & $\phi_{R N}^{G H}(u)=e^{i \Delta u t}\left(\frac{\alpha^{2}-\beta^{2}}{\alpha^{2}-(\beta+i u)^{2}}\right)^{\lambda t / 2} \frac{K_{\lambda}\left(\delta \sqrt{\alpha^{2}-(\beta+i u)^{2}}\right)^{t}}{K_{\lambda}\left(\delta \sqrt{\alpha^{2}-\beta^{2}}\right)}$ \\
NIG & $\phi_{R N}^{N I G}(u)=\exp \left\{i \Delta u t-\delta t\left(\sqrt{\alpha^{2}-(\beta+i u)^{2}}-\sqrt{\alpha^{2}-\beta^{2}}\right)\right\}$ \\
GIG & $\phi_{R N}^{G I G}(u)=e^{i \Delta u t}\left(1-\frac{2 i u}{\gamma^{2}}\right)^{\lambda t / 2} \frac{K_{\lambda}\left(\delta \gamma \sqrt{1-2 i u \gamma^{-2}}\right)^{t}}{K_{\lambda}(\delta \gamma)}$ \\
VG & $\phi_{R N}^{V G}(u)=e^{i \Delta u t}\left(1-i u v \theta+\frac{1}{2} \sigma^{2} u^{2} v\right)^{\frac{-t}{v}}$ \\
\hline
\end{tabular}

Then, the risk-neutral stock price process can be formulated using equation (31) and equation (32) for the characteristic function of the risk-neutral stock price process.

\subsection{European Options Pricing}

Herewith, the pricing of the European is focused on a given market model, when the payoff function is only a function of the terminal stock price. 


\subsubsection{Pricing through the Density Function}

Let $K$ be a strike price, $S_{T}$ be an asset price and $T$ be an expiration of a contingent claim. The value plain vanilla call option prices at time 0 are given by the expectation of the payoff under the martingale measure $\mathbb{Q}$ is given by;

$$
C\left(t, S_{t}\right)=\exp (-r T) E^{\mathbb{Q}}\left[\left(S_{T}-K\right)^{+}\right] .
$$

when $\mathbb{Q}$ is the Esscher transform equivalent martingale measure, the above expectation can be written as;

$$
\exp (-r T) S_{0} \int_{c}^{\infty} f_{T}^{\left(\theta^{*}+1\right)}(x) d x-\exp (-r T) K \int_{c}^{\infty} f_{T}^{\left(\theta^{*}\right)}(x) d x,
$$

where $c=\ln \left(K / S_{0}\right)$.

\subsubsection{Option Pricing with Fast Fourier Transformation (FFT)}

In general, typically to find an analytical solution in Lévy process models like a Black-Scholes (BS) formula. Carr and Madan (1998) showed how to use the FFT to value options efficiently based on the characteristic function of the asset. The BS model with Fourier transform pricing methodology is presented first to illustrate the idea clearly and simply. A brief derivation is given for this method is the simplest case which needs to be extended to allow for a FFT to be implemented.

Consider the European call option with the asset price process $S_{T}$ time maturity $T$, strike price $K, s_{T}=\ln S_{T}$, $k=\ln K, q_{T}(s)$ is the risk-neutral density of $s_{T}$ and $C_{T}(k)$ is the European call option value at time $\mathrm{T}$ with strike $\exp (k)$. The characteristic function of $s_{T}$ is defined by;

$$
\phi_{T}(u)=E\left[\exp \left(i u s_{T}\right)\right]=\int_{-\infty}^{\infty} e^{i u s} q_{t}(s) d s .
$$

At the expiration date $T$, the option value $C_{T}$ which is related to the risk-neutral density $q_{T}$ is given by;

$$
C_{T}(k)=e^{-r T} E\left[\left(S_{T}-K\right)^{+}\right]=\int_{k}^{\infty} e^{-r T}\left(e^{s}-e^{k}\right) q_{T}(s) d s .
$$

Here $C_{T}(k)$ is not square-integrable because $C_{T}(k) \rightarrow S_{0}$ as $k \rightarrow \infty$ so that $K \rightarrow 0$. To obtain a square integral function Carr and Madan (1998) suggested considering the modified price $c_{T}(k)$ given by;

$$
c_{T}(k)=e^{\alpha k} C_{T}(k) \text {, }
$$

for some $\alpha \in \mathbb{R},(>0)$. Here, Carr and Madan(1998) suggested choosing the decay rate parameter $\alpha$ as $\alpha \approx 0.25$, while Schoutens (2003) suggests $\alpha \approx 0.75$. The value of $\alpha$ affects the speed of convergence. The Fourier transform of $\mathrm{c}_{\mathrm{T}}(\mathrm{k})$ is given by;

$$
\psi_{T}(v)=\int_{-\infty}^{\infty} e^{i v k} c_{T}(k) d k,
$$

and corresponding inverse Fourier transform of $C_{T}(k)$;

$$
C_{T}(k)=\frac{e^{-\alpha k}}{2 \pi} \int_{-\infty}^{\infty} e^{-i v k} \psi_{T}(v) d v=\frac{e^{-\alpha k}}{\pi} \int_{0}^{\infty} e^{-i v k} \psi_{T}(v) d v
$$

Then, $\psi_{T}(v)$ can be computed in terms of the characteristic function of the risk-neutral density of $s_{T}=\ln S_{T}$.

$$
\begin{aligned}
\psi_{T}(v) & =\int_{-\infty}^{\infty} e^{i v k} \int_{k}^{\infty} e^{\alpha k} e^{-r T}\left(e^{s}-e^{k}\right)^{+} \mathrm{q}_{\mathrm{T}}(s) d s d k \\
& =\int_{-\infty}^{\infty} e^{-r T} \mathrm{q}_{\mathrm{T}}(s) \int_{-\infty}^{s}\left(e^{s+\alpha k}-e^{(1+\alpha) k}\right) e^{i v k} d k d s ;
\end{aligned}
$$

by Fubini and $\left|\psi_{T}(v)\right|<\infty$

$$
\begin{gathered}
=\int_{-\infty}^{\infty} e^{-r T} \mathrm{q}_{\mathrm{T}}(s)\left[\frac{e^{s(1+\alpha+i v)}}{\alpha+i v}-\frac{s(1+\alpha+i v)}{1+\alpha+i v}\right] d s \\
\psi_{T}(v)=e^{-r T} \frac{\phi_{T}(v-(1+\alpha) i)}{\alpha^{2}+\alpha-v^{2}+i(2 \alpha+1) v} .
\end{gathered}
$$

European option price can be calculated by substituting (41) into (40) as follows;

$$
C_{T}(k)=\frac{e^{-\alpha k}}{\pi} \int_{0}^{\infty} e^{-i v k} e^{-r T} \frac{\phi_{T}(v-(1+\alpha) i)}{\alpha^{2}+\alpha-v^{2}+i(2 \alpha+1) v} d v .
$$

\subsection{Numerical Implementation}

Fast Fourier transformation (FFT) is an efficient algorithm to compute the following sum:

$$
F(k)=\sum_{i=1}^{N} f_{j} \exp \left(\frac{-i 2 \pi}{N}(j-1)(k-1)\right)
$$


where $\mathrm{N}$ is usually a power of 2. FFT is a commonly employed discrete approximation technique of Fourier transform used to reduce computational labor. Suppose that we want to approximate the inverse Fourier transform of a function $\mathrm{C}_{\mathrm{T}}(\mathrm{k})$ in (40) with discrete FFT. Then this integrand should be truncated and discretized by:

$$
C_{T}(k) \approx \frac{\exp (-\alpha k)}{\pi}\left(\sum_{i=1}^{N} e^{-i v_{j} k} \psi_{T}\left(v_{j}\right) \eta\right)
$$

with the following conventions and parameter values to apply equation (46) (as suggested by Carr and Madan, 1998); $v_{j}=\eta(j-1), N=2^{12}=4096, a=N \eta=600, b=\frac{N \lambda}{2}, k_{u}=-b+\frac{2 b}{N}(u-1), \lambda \eta=\frac{2 \pi}{N}$. Here a is the upper limit for the integration, while $k_{u}$ are a vector with $N$ values of $k$ and $b$ sets a bound on the $\log$ strike to range between $-b$ and $b$. Therefore, formula (44) can be written a;

$$
C_{T}(k) \approx \frac{\exp \left(-\alpha k_{u}\right)}{\pi}\left(\sum_{j=1}^{N} e^{-i \lambda \eta(j-1)(u-1)} e^{i b v_{j}} \psi_{T}\left(v_{j}\right) \eta\right) .
$$

Carr and Madan suggest using Simpson's weighting rule to obtain an accurate integration with large $\eta$ (Note 4). The price formula can be written as:

$$
C_{T}(k) \approx \frac{\exp \left(-\alpha k_{u}\right)}{\pi} \frac{\eta}{3} \sum_{j=1}^{N} e^{-\frac{i 2 \pi}{N}(j-1)(u-1)} e^{i b v_{j}} \psi_{T}\left(v_{j}\right)\left(3+(-i)^{j}-\delta_{j-1}\right),
$$

where $\delta_{n}$ is the Kronecker delta function.

\section{Model Calibration}

\subsection{Data Description}

For the empirical study, daily prices of physically settled Gold futures and American-style options written on these futures contracts traded at the COMEX were considered. The price taken as the daily price is the last trading price of the day as quoted in U.S. dollars and cents per troy ounce. Further, the Price for gold futures is for the nearest expiration contract and the data was obtained from Bloomberg. Data on futures prices span the period from January 2, 2007, to December 27, 2017, whereas the available options data set spans the period January 3, 2017, to December 27, 2017, and comprises 251 trading days. There are several maturities are available in each and every trading day. Call options and the corresponding futures contracts are available with maturities in each calendar month. While trading in the futures contract ceases three business days prior to the first day of the delivery month, trading in the options written on this futures contract ends on the business day before the last trading day of the futures.

Following filters are applied to options data set before calibration:

- Keep options whose maturity time less than 6 months due to volume decreases substantially with expiration dates.

- Exclude the very near-term futures options, which are less than $1(<1)$. (These options have early exercise opportunities at most four times)

- $\quad$ Remove deep-in-the-money $(\mathrm{S} / K>1.2)$ (ITM) and deep out-of-the-money $(\mathrm{S} / K<0.8)$ (OUT) call options. Therefore, we select the options close to at-the-money, ie. moneyness $(\mathrm{S} / K)$ in between 0.8 and

$1.2(0.8<\mathrm{S} / K<1.2)$. These options are often illiquid or with a price next to zero.

After applying all filters, the data used consists of a total of 96,567 call prices to calibrate over the period. The constant three month T-bill is used as a risk-free rate.

\subsection{Price Process and Calibration Procedure}

These American style option prices are converted to European style option prices using Barone-Adesi and Whaley (1987) quadratic approximation method to adjust for the early exercise premium. Therefore, the European option methodology can be applied to calibrate the options based on the FFT method. Choosing a risk-neutral model such as to reproduce the prices of traded options is known as model calibration: given market prices $\left(C_{i}^{\text {market }}\right)_{i \in I}$ at $t=0$ for a set of benchmark option with different strikes $K_{i}$ and maturities $T_{i}$, one looks for a risk-neutral model $\mathbb{Q}$ which correctly prices these options: 


$$
\forall i \in I, C_{i}^{\text {market }}\left(\hat{\vartheta} ; S_{T_{i}}, K_{i}, T\right)=e^{-r T} \mathbb{E}^{\mathbb{Q}}\left[\left(S_{T_{i}}-K_{i}\right)^{+} \mid \mathcal{F}_{t}\right]
$$

The pricing problem is concerned with computing values of the option given model parameters; i.e direct problem or indirect problem. A direct problem (simple and older one) can be formulated using equation (47). European option prices $C_{i}^{\text {market }}$ across different strikes $\mathrm{K}_{\mathrm{i}}$ are calculated given a vector of parameters $\widehat{\vartheta}$ and variables such as strikes $\mathrm{K}_{\mathrm{i}}$ and a maturity $\mathrm{T}$. (where $\hat{\vartheta}$ be a vector of parameters to be identified for a given Levy process) Here, our interest is in backing out parameters describing risk-neutral dynamics from observed option prices, ie. inverse problem. Cont and Tankov (2004) pointed out that it is not easy to find an exact solution and the inverse problem is ill-posed. Because there may be many pricing models that generate the same prices for the benchmark options thus the solution of the inverse problem is not necessarily unique. Another problem is, of course, the computation of a solution for the inverse problem, for which efficient and stable algorithms are needed.

Thus, a calibration problem can be expressed as a nonlinear least-squares problem in practice for a given call option prices $C_{i}^{\text {model }}$ and find a parameter vector $\widehat{\vartheta}$ such that;

$$
\underset{\hat{\vartheta}}{i n f}\left\|C_{i}^{\text {market }}-C_{i}^{\text {model }}\right\|^{2} \text {. }
$$

As mentioned by Tankov, this problem has some difficulties such that; it is hard to identify the optimal model due to a finite number of market data used to solve this, the equation (47) has many local minima due to function is non-convex. Cont and Tankov reformulate the least square problem (47) to overcome these technical difficulties. They use the regularization method to approximate the solution to remove pricing errors.

Also, the smallest relative entropy with respect to a given prior measure is used to take care of its lack of identification problem. However, in our paper, we check the relative errors with the infinity norm as the allowing tolerance 1.E-6, and the gradient norm as well. Secondly, in order to reduce the market pricing noise, we use the common method of eliminating data points that are deep out of the money and deep in the money. Now our calibration problem is set up as;

$$
\hat{\vartheta}=\arg \underset{\vartheta}{\min } \sum_{i==1}^{N}\left(C_{i}^{\text {model }}\left(\hat{\vartheta} ; S_{T_{i}}, K_{i}, T\right)-C_{i}^{\text {market }}\right)^{2}
$$

for some finite $\mathrm{N}$ call options in the market.

\section{Numerical Results}

According to the data and procedure mentioned earlier, the parameters underlying the Black-Scholes (BS), GH, NIG, GIG and VG models were calibrated for Gold options. Each model is calibrated for each maturity separately. For the sake of simplicity and to focus on the essence of the stochastic behavior of the asset, the risk-free interest rate is fixed as $10 \%$ and dividend yield to zero.

\subsection{Parameter Calibrated}

In Table 3, reports the time-averaged parameters with their standard errors of each model, because each parameter is calibrated with each maturity separately for the sample period. Note that, in NIG's $\mu$ is set to zero due to redundant.

Table 3. Lévy Model parameters estimation (mean correcting)

\begin{tabular}{lcccc}
\hline Model & \multicolumn{3}{c}{ Parameter Estimated with Standard Errors } \\
\hline BS & $\sigma=0.1734(0.0356)$ & $\alpha=8.4321$ & $\beta=2.2125$ & $\delta=0.2453$ \\
GH & $\lambda=0.891$ & $(9.581)$ & $(2.213)$ & $(0.165)$ \\
& $(0.095)$ & $\beta=2.3412$ & $\delta=0.1041$ & \\
NIG & $\alpha=12.4183$ & $(2.651)$ & $(0.115)$ & \\
& $(10 . .231)$ & $\delta=2.1571$ & $\gamma=1.5487$ & \\
GIG & $\lambda=0.9643$ & $(1.954)$ & $(1.012)$ & \\
& $(0.856)$ & $\sigma=0.2814$ & $v=0.4617$ & \\
VG & $\theta=0.1354$ & $(0.017)$ & $(0.239)$ & \\
& $(0.065)$ & & \\
\hline
\end{tabular}

Standard errors are included within the parentheses. 
The results of the five calibrated models for call options are visualized as the time to maturity (from one month to six-month maturity) for all the strikes (1500- 2100) on 01.10.2012, in Figure B.1 through Figure B.5 for the BS, GH, NIS, GIG and VG respectively (see Appendix B). In each figure, circles are the market prices and the plus signs are the model prices. It is difficult to say which model works better than others by looking at each and every figure.

Therefore, in Figure 1, all calibrated models are compared with market Gold options to get an overall impression for one maturity; that is to say one rage of strikes (2 months to maturity, on October 2017 options). According to Figure 1, the NIG model gives a very closer fit than other models. However, as we expected BS model is far from the market data from the other models (It illustrates the well-known bias of the BS model which is to underprice the ITM calls and overprice the OTM calls) (Note 5). Furthermore, the GH model is the second, the VG model is the third and the GIG model is the fourth. Still, it is difficult to identify which model gives better best. Some are close to one and another. There are differences between in-the-money and out-the-money too. However, some models can work better on a dataset and worse on another. For clarification, the sum of squared errors (SSE) is calculated and listed in Table 4. A similar result is generated as seen in the graphs.

Table 4. Sum of squared errors (SSE) for the sample period

\begin{tabular}{cccccc}
\hline Models & BS & GH & NIG & GIG & VG \\
\hline SSE & 198.541 & 1.354 & 0.985 & 12.654 & 2.197 \\
\hline
\end{tabular}

For more comparative purposes, in-sample pricing performance and out-of-sample performance are computed under several global measures of fit in the next section.

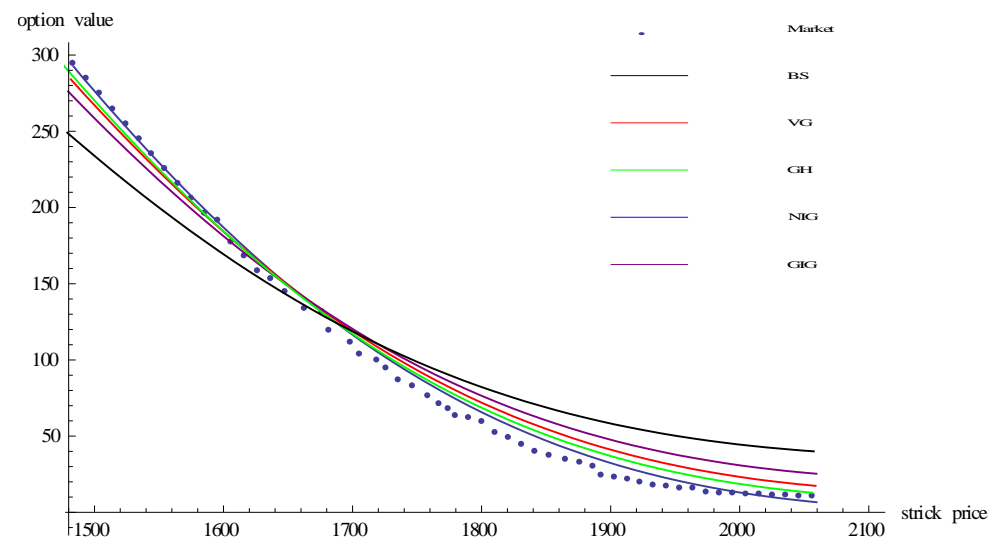

Figure 1. Comparison between market Gold option prices and results of calibrated five models

\subsection{Pricing Performance}

In order to see the pricing performance of the chosen models, the following errors are performed: average pricing error (APE), average absolute error (AAE), average relative percentage error (ARPE) and root mean square error (RMSE).

$$
\begin{aligned}
A P E & =\frac{\sum_{i=1}^{N}\left|C_{i}^{\text {market }}-c_{i}^{\text {model }}\right|}{\sum_{i=1}^{N} C_{i}^{\text {market }}} \\
A A E & =\sum_{i=1}^{N} \frac{\left|c_{i}^{\text {market }}-C_{i}^{\text {model }}\right|}{N} \\
A R P E & =\frac{1}{N} \sum_{i=1}^{N} \frac{\left|c_{i}^{\text {market }}-C_{i}^{\text {model }}\right|}{\sum_{i=1}^{N} C_{i}^{\text {market }}} \\
R M S E & =\sqrt{\sum_{i=1}^{N} \frac{\left(c_{i}^{\text {market }}-C_{i}^{\text {model }}\right)^{2}}{N}}
\end{aligned}
$$

Time-averaged APE, AAE, ARPE, and RMSE are listed in Table 5. 


\subsubsection{In-Sample Pricing Performance}

The time-averaged APE, AAE, ARPE, and RMSE are reported in Table 5. NIG model lists the lowest pricing error under APE and RMSE and GH model reports the lowest pricing error under AAE and ARPE while the BS model gives the highest errors under all criteria. Therefore, we are unable to decide the better-fitted model to Gold options prices according to in-sample pricing performance. However, there are more parameters in the GH model (four parameters) than the NIG model (three parameters). When a number of parameters increases, the fitted model is more closed with market data. It can caught-up more variability in the market.

Table 5. In-sample pricing Errors for the selected Lévy models

\begin{tabular}{ccccccc}
\hline Models & BS & GH & NIG & GIG & VG & Decision \\
\hline APE & 0.3975 & 0.0121 & 0.0098 & 0.0265 & 0.0220 & Min. in NIG \\
AAE & 7.4543 & 1.0632 & 1.1512 & 2.3120 & 1.7553 & Min. in GH \\
ARPE & 0.4193 & 0.0483 & 0.0495 & 0.0994 & 0.0746 & Min. in GH but almost close to NIG \\
RMSE & 1.0244 & 0.0126 & 0.0091 & 0.1501 & 0.0426 & Min. in NIG \\
\hline
\end{tabular}

Therefore, it is necessary to discuss the out-of-sample performance among Lévy models versus the Black-Scholes model. Moreover, if the extra parameters of the jump Lévy models are redundant, they overfit the data and may produce larger out-of-sample fitting errors.

\subsubsection{Out-of-Sample Pricing Performance}

It is well known that in-sample fit alone is not enough to guarantee the predictability problem for empirical data. Also, we may not determine which model is the best fit for specific data. To check this reliability, we conduct the out-of-sample performance. We used the Bakshi, Cao, and Chen (1997) procedure in the following manner.

Let $t$ denote today price. Firstly, on yesterday $(t-1)$, the parameters of each Lévy model are recovered using yesterday's call prices and variables. Secondly, today's model prices are computed using yesterday's calibrated parameters $\widehat{\vartheta_{\mathrm{t}-1}}$ and today's variables. Thirdly, using today's model prices and today's call prices, the absolute pricing error is obtained following the equation:

$$
\text { Absolute Pricing Error }=\left|C_{t}^{\text {market }}-C_{t}^{\text {model }}\left(\widehat{\vartheta_{t-1}} ; S_{T_{i}}, K_{i}, T-t\right)\right| \text {. }
$$

This procedure is repeated every day across all the call strike prices. Table 6 reports the out-of-sample absolute pricing errors per call option. Also, we hold the same filter on out-of-sample observations and all parameters for the numerical schemes are kept. Similarly, we investigate the pricing performance as we have done for in-sampling. Overall, the NIG process outperforms the other models. The B-S model's absolute error was, on average, $12 \%$ greater than those of the NIG process.

Table 6. Out-of-sample average absolute error per call option

\begin{tabular}{ccccccc}
\hline Models & BS & GH & NIG & GIG & VG & Decision \\
\hline APE & 5.638 & 0.501 & 0.486 & 0.974 & 0.721 & Min in NIG \\
\hline
\end{tabular}

Based on all the selection criteria, the NIG model shows a better fit than other models for Gold Options.

\section{Conclusion}

Today Gold futures markets are volatile. Therefore, option prices do not support the Black-Scholes world of constant volatility and the lognormal distribution of asset prices at options expiration. Hence, it is necessary to add the jump process to the model. Lévy's process fame-works can capture these difficulties in the real market.

In the first part of this study, some exponential Lévy processes used for option pricing were introduced with their foremost mathematical properties. Then, option pricing with Fourier Transform methods was calculated based on the knowledge of the characteristic function, performing the FFT algorithm. Finally, the model parameters were calibrated to option market prices with the non-linear least-squares method.

The Black-Scholes model with four different Lévy models (Generalized Hyperbolic process, Normal Inverse Gaussian Process, Generalized Inverse Gaussian Process and Variance Gamma Process) are calibrated to the Gold futures options and compares their pricing performance under in-sampling and out-of-sampling. The result shows that jump Lévy models drastically outperform the classic Black-Scholes model in pricing. Moreover, the NIG 
model provides better performance than all other Lévy models under all selection criteria for Gold options. Further, this study can be extended to other option markets to verify the selected model for option pricing.

\section{References}

Aballero, M., \& Chaumont, L. (2006). Conditioned stable Lévy processes and the Lamperti representation. Journal of Applied Probability, 43(4), 967-983. https://doi.org/10.1017/S0021900200002369

Bakshi, G., Cao, C., \& Chen, Z. (1997). Empirical Performance of Alternative Option Pricing Models. Journal of Finance, 52(1997), 2003-49. https://doi.org/10.1111/j.1540-6261.1997.tb02749.x

Barndorff-Nielsen, O. E. (1977). Exponentially decreasing distributions for the logarithm of particle size, Proceedings of the Royal Society London A 353, 401-419. https://doi.org/10.1098/rspa.1977.0041

Barndorff-Nielsen, O. E. (1995). Normal Inverse Gaussian Processes and the Modelling of Stock Returns. Research Report 300, Department of Theoretical Statistics, University of Aarhus.

Barndorff-Nielsen, O. E. (2001a). Non-Gaussian Ornstein-Uhlenbeck-based models and some of their uses in financial economics. Journal of the Royal Statistical Society, B 63, 167-241. https://doi.org/10.1111/14679868.00282

Barone-Adesi, G., \& Whaley, R. E. (1987). Efficient analytic approximation of American option values. Journal of Finance, 42, 301-320. https://doi.org/10.1111/j.1540-6261.1987.tb02569.x

Baur, D., \& Lucey, B. (2010). Is gold a hedge or a safe haven? An analysis of stocks, bonds, and gold. The Financial Review, 45, 217-229. https://doi.org/10.1111/j.1540-6288.2010.00244.x

Black, F., \& Scholes, M. (1973). The Pricing of Options and Corporate Liabilities. Journal of Political Economy, 81, 637-654. https://doi.org/10.1086/260062

Carr, P. H., Geman, D. M., \& Yor, M. (2003). Stochastic Volatility for Lévy Processes. Mathematical Finance, 13(3), 345-382. https://doi.org/10.1111/1467-9965.00020

Carr, P., \& Madan, D. H. (1998). Option valuation using the fast Fourier transform. Journal of Computational Finance, 2, 61-73. https://doi.org/10.21314/JCF.1999.043

Carr, P., Geman, H., Madan, D., \& Yor, M. (2007). Self-decomposability and option pricing. Mathematical Finance, 17(1), 31-57. https://doi.org/10.1111/j.1467-9965.2007.00293.x

De Finetti, B. (1929). Sulle funzioni ad incremento aleatorio. Rend. Acc. Naz. Lincei., 10, 163-168.

Eberlein, E. (2007). Jump-type Lévy processes. Working Paper, University of Freiborg.

Eberlein, E., \& Hammerstein, E. A. V. (2004). Generalized hyperbolic and inverse Gaussian distributions: limiting cases and approximation of processes. Seminar on Stochastic Analysis, Random Fields and Applications IV, Progress in Probability, 58, 221. https://doi.org/10.1007/978-3-0348-7943-9_15

Gerber, H. U., \& Shiu, E. S. W. (1994). Option Pricing by Esscher Transforms. Transactions of the Society of Actuaries, 46, 99-191.

Halgreen, C. (1977). Self-decomposability of the generalized inverse Gaussian and hyperbolic distribution functions. Z.Wahrschein. Verw.Gebiete, 47, 13-18. https://doi.org/10.1007/BF00533246

Heyde, C. C., \& Leonenko, N. N. (2005). Student processes. Advances in Applied Probability, 37(2), 342-365. https://doi.org/10.1239/aap/1118858629

Ken-Iti, S. (1999). Lévy processes and infinitely divisible distributions. Cambridge, UK: Cambridge University Press.

Kumari, S. N., \& Tan, A. (2013). Asset return distribution and its application to gold future index. GSTF Journal of Mathematics, Statistics and Operation Research, 2(1), 72-76. https://doi.org/10.5176/2251-3388_2.1.34

Kyprianou, A. E. (2014). Fluctuations of Lévy Processes with Applications (2nd ed.). Springer. https://doi.org/10.1007/978-3-642-37632-0

Lawrence, C. (2003). Why is gold different from other assets? An empirical investigation (World Gold Council, London).

Lucey, B., \& Tully, E. (2006). Seasonality, risk and return in daily comex gold and silver 1980-2002. Appl. Financial Econ., 16, 519-533. https://doi.org/10.1080/09603100500386586

Madan, D. B., Carr, P., \& Chang, E. (1998). The Variance Gamma Process and Option Pricing. European Finance 
Review, 2, 79-105. https://doi.org/10.1023/A:1009703431535

Marc, C. (2010). An Introduction to Implied Lévy Volatility with Applications. Master thesis, August 30, 2010.

Necula, C. (2009). Modeling Heavy-Tailed Stock Index Returns Using the Generalized Hyperbolic Distribution. Romanian Journal of Economic Forecasting, 10(2), 118-131.

Petroni, N. C. (2008). Self-decomposability and self-similarity: A concise primer. Physica A, 387, 1875-1894. https://doi.org/10.1016/j.physa.2007.11.036

Sato, K. I. (1991). Self-similar processes with independent increments. Prob. Theor. Related Fields, 89, 285-300. https://doi.org/10.1007/BF01198788

Sato, K. I. (1999). Lévy processes and infinitely divisible distributions. Cambridge: Cambridge Univ. Press.

Schoutens, W. (2003). Lévy Process in Finance: Pricing Financial Derivatives. New York: John Wiley \& Sons. https://doi.org/10.1002/0470870230

Scott-Ram, R. (2002). Managing Portfolio Risk with Gold. World Gold Council, London.

\section{Notes}

Note 1 . Let $\left(Y_{n} ; n=1,2, \ldots\right)$ is a sequence of independent random variables and $S_{n}=\sum_{i=1}^{n} Y_{i}$ denotes their sum. Suppose that, there exist centering constants $a_{n} \in \mathbb{R}$ and scaling constants $b_{n}>0$, such that the distribution of $b_{n} S_{n}+a_{n}$ converges to the distribution of some random variable $X$. Then we say that random variable $X$ is a member of class $L$. As explained above, we shortly can say that, if a random variable $X$ has the same distribution of the limit of some sequence of normalized sums of independent random variables, random variable $X$ has a distribution of class $L$ by Carr, Geman, Madan and Yor (2007).

Note 2 . The density function of the $\operatorname{IG}(\delta, \gamma)$ law is given by;

$f_{I G}(x ; \delta, \gamma)=\frac{\delta}{\sqrt{2 \pi}} \exp (\delta \gamma) x^{-\frac{3}{2}} \exp \left(-\frac{1}{2}\left(\frac{\delta^{2}}{x}+\gamma^{2} x\right)\right)$, for $x>0$.

Note 3. The new distribution is formed by adding drift or location parameter $(m \in \mathbb{R})$ without changing the original one. This extension does not influence the infinite divisibility property or self-decomposability. The new distribution has a characteristic function $\bar{\phi}$ in term of the original characteristic function $\phi: \phi(u)=$ $\bar{\phi}(u) \exp ($ ium).

Note 4. Here combine of fine integration grid with a wide enough region for strikes are not possible, as if we choose a too small $\eta$ we get a fine integration grid but few strikes lying in the region.

Note 5. ITM (in-the-money), the moneyness (K/S) is less than one and OTM (out-the-money) the moneyness (K/S) is greater than one. In Figure 3.1, the price $\mathrm{S}$ consider as $\$ 1680$ and $\mathrm{K}$ is the rage between 1490 to 2100 for the increment 10 .

\section{Appendix A. Risk-Neutral Variance Gamma Characteristic Function}

The characteristic function of the Variance Gamma process under the measure $\mathbb{P}$ is given by:

$$
\phi_{V G}(u)=\left(1-i u \theta v+\frac{1}{2} \sigma^{2} v u^{2}\right)^{-\frac{t}{v}},
$$

and this can be re-written as;

$$
\phi_{V G}(u)=\exp \left[-\frac{t}{v} \ln \left(1-i u \theta v+\frac{1}{2} \sigma^{2} v u^{2}\right)\right] .
$$

This is a characteristic exponent of VGP and it can be split into two parts: a drift part $\mu(u)=0$ and a non-drift part $\varphi(u)=-\frac{t}{v} \ln \left(1-i u \theta v+\frac{1}{2} \sigma^{2} v u^{2}\right)$.

The drift part can be re-written under $\mathrm{RN}$ measure as:

$$
\mu^{R N}(u)=i\left[r-\frac{\varphi(-i)}{t}\right] u t
$$




$$
\begin{aligned}
& =i\left[r+\frac{1}{v} \ln \left(1-\theta v-\frac{1}{2} \sigma^{2} v\right)\right] u t \\
& =i \Delta u t
\end{aligned}
$$

where $\Delta$ is a RN drift; $\Delta=r+\frac{1}{v} \ln \left(1-\theta v-\frac{1}{2} \sigma^{2} v\right)$.

Therefore, the RN characteristic function of the VGP is formulated as:

$$
\phi_{V G}^{R N}(u)=e^{\varphi(u)+\mu^{R N}(u)}
$$

$$
\phi_{V G}^{R N}(u)=e^{i \Delta u t}\left(1-i u \theta v+\frac{1}{2} \sigma^{2} v u^{2}\right)^{-\frac{t}{v}} .
$$

Thus, the risk-neutral stock price process is given by:

$$
S_{t}=S_{0} \exp \left\{\Delta t+X_{t}^{V G}\right\},
$$

where $X_{t}^{V G}$ is a VGP.

Let $S_{0}=1$, for simplicity of calculation, then $\log S_{0}=0$.

This gives the risk-neutral exponential $\mathrm{VG}$ process as;

$$
\log S_{t}=r t+\frac{t}{v} \ln \left(1-\theta v-\frac{1}{2} \sigma^{2} v\right)+X_{t}^{V G}
$$

and this is the same equation in (34). Therefore, the characteristic function of the risk-neutral log stock price of Variance gamma process, $\phi_{V G}^{R N}(u)$, can be used to price European options using equation (41) for known parameters of $\theta, v$ and $\sigma$.

\section{Appendix B. The plot of Market Prices Vs Model Prices}

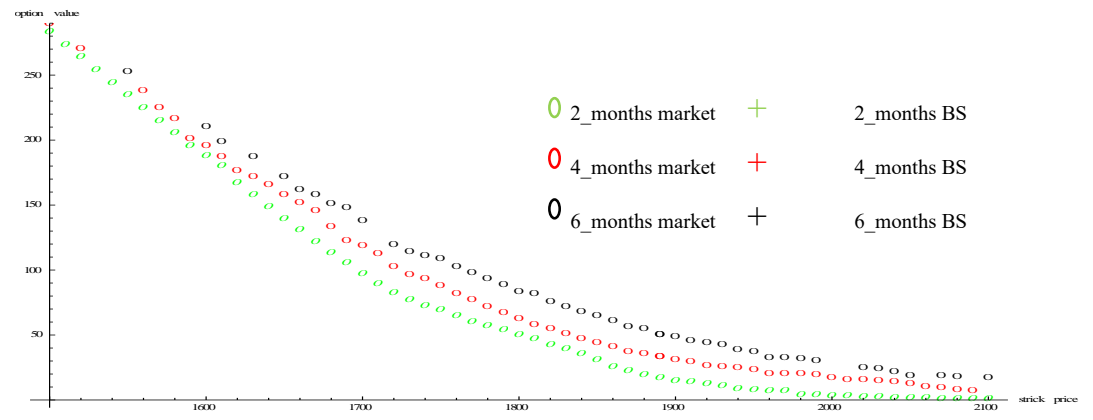

Figure B.1. Option market prices on 01/10/2017for Gold options (rings) and prices given by the Black-Scholes model pluses) for six different maturities $(2,4 \& 6$ months $)$

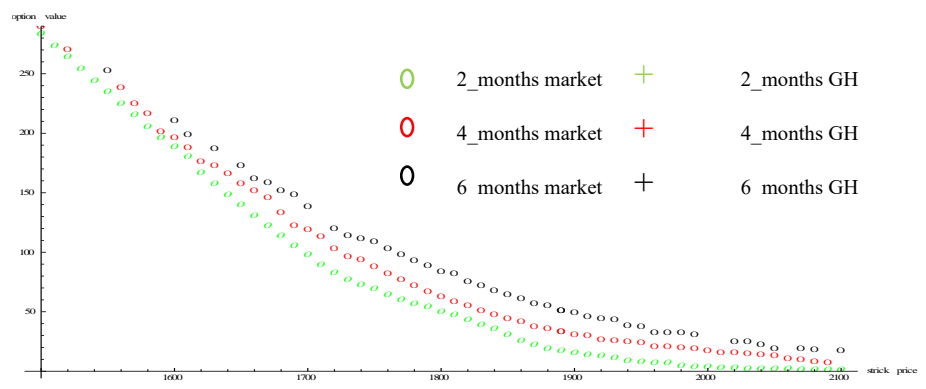

Figure B.2. Option market prices on 01/10/2017 for Gold options (rings) and prices given by the GeneralizedHyperbolic model pluses) for six different maturities $(2,4 \& 6$ months $)$ 


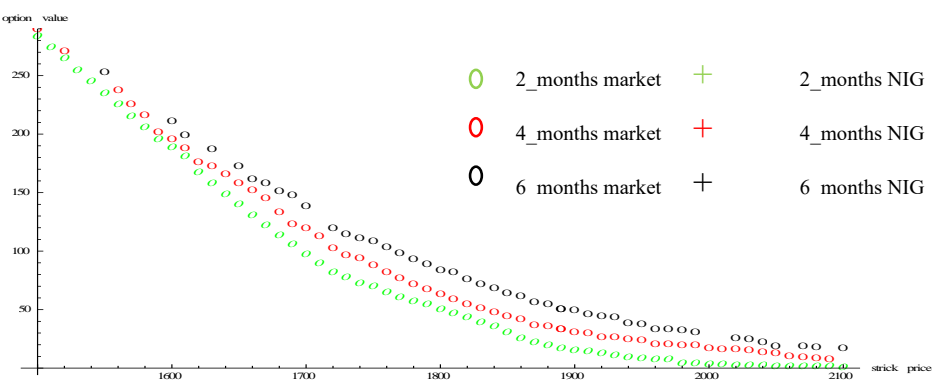

Figure B.3. Option market prices on 01/10/2017 for Gold options (rings) and prices given by the Normal Inverse Gaussian model (plus) for six different maturities $(2,4 \& 6$ months)

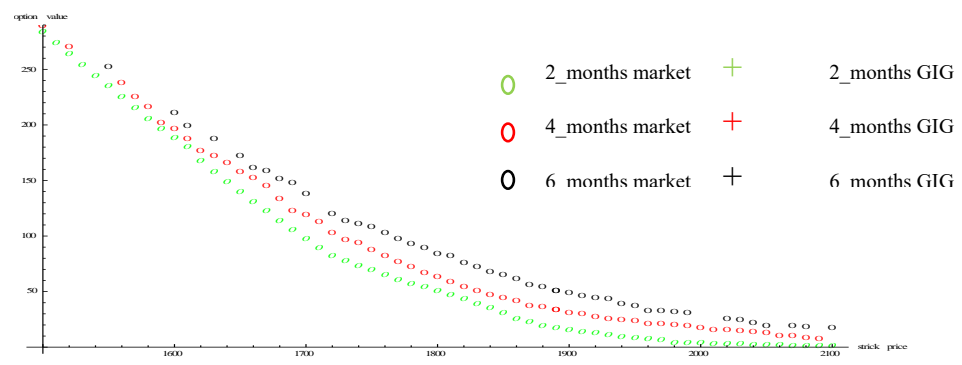

Figure B.4. Option market prices on 01/10/2017 for Gold options (rings) and prices given by the Generalized Inverse Gaussian model (pluses) for six different maturities $(2,4 \& 6$ months)

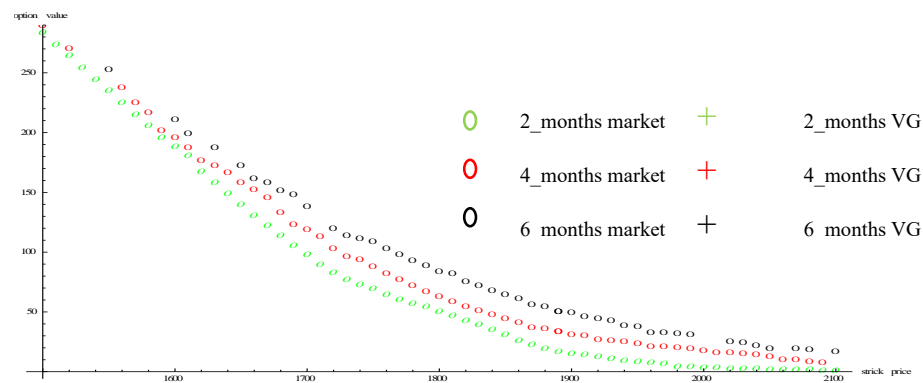

Figure B.5. Option market prices on 01/10/2017 for Gold options (rings) and prices given by the VarianceGamma model (pluses) for six different maturities $(2,4 \& 6$ months)

\section{Copyrights}

Copyright for this article is retained by the author(s), with first publication rights granted to the journal.

This is an open-access article distributed under the terms and conditions of the Creative Commons Attribution license (http://creativecommons.org/licenses/by/4.0/). 